\title{
Stability After Anterior Lumbar Fusion with Interbody Cages: A Radiostereometric Evaluation
}

\author{
Paul Axelsson, Ragnar Johnsson and Björn Strömqvist*
}

Department of Orthopedics, Lund University Hospital, SE-221 85 Lund, Sweden

\begin{abstract}
Background: Interbody cage stabilization as a stand-alone procedure in lumbar spine fusion is questioned due to inconsistent mechanical effects registered in human cadaveric studies. Even segmental mobility exceeding the normal range of motion is described after cage implantation. For the anterior interbody cage fusion, this potential, undesired destabilization is explained by the resection of the anterior longitudinal ligament included in the surgical procedure.

Patients and Methods: Four patients with degenerative disc disease and long-standing lumbar pain had an anterior lumbar fusion using threaded interbody cages. Five segments with defined preoperative mobility measured by radiostereometry (RSA) were treated. The postoperative mobility effects after cage stabilization were assessed by repeat RSA 1, 6 and 12 months after surgery and related to the preoperative findings.

Results: Before surgery, the lumbar segments aimed for fusion were all mobile with a significant sagittal mobility, range 1.3 to $3.1 \mathrm{~mm}$. One month after cage implantation, the intervertebral mobility was reduced in all segments and fully abolished in three. Persisting but minor sagittal translations were seen in two segments. One of these turned fully stable at the one-year follow-up.

Interpretation: According to our study, the anterior interbody cage fusion has the biomechanical potential to provide immediate segmental stabilization. The technique can be used as a stand-alone procedure without supplementary instrumentation. The adequate mechanical properties justify extended clinical studies on outcome and complications to evaluate the anterior interbody cage stabilization as instrument in lumbar fusion.
\end{abstract}

\section{INTRODUCTION}

Favourable outcome described for the anterior interbody cage fusion as a stand-alone procedure [1] has later been contradicted by a discouraging incidence of pseudarthrosis reported for the technique [2]. Human cadaveric studies imply that the anterior interbody cage stabilization is incomplete especially in extension and supplementary instrumentation is often recommended to improve the fixation in all directions [3]. The biomechanical consequences of the cage implantation are inconsistent and even surgical destabilization with increased segmental mobility after surgery is registered in vitro [4]. This paradoxical effect could be explained by the resection of the anterior longitudinal ligament included in the surgical procedure.

Radiostereometry (RSA) [5] can measure spinal intervertebral mobility in vivo with a very high accuracy [6, 7]. The technique has prior been applied to assess the biomechanical characteristics for the posterior instrumentation which mostly induces immediate total stabilization if the surgery does not include broad decompression including facetectomy [8]. In the current study, patients with disc degenerative disease/segmental pain

*Address correspondence to this author at the Department of Orthopedics, Lund University Hospital, SE-221 85 Lund, Sweden; Tel: 046-46-171000; Fax: 046-46-130732; E-mail: bjorn.stromqvist@med.lu.se and preoperatively defined ranges of mobility over the painful segment, were examined with radiostereometry through the postoperative course after anterior interbody cage stabilization. Postoperative intervertebral mobility was related to the preoperative findings with the aim to evaluate whether the effect of the anterior interbody cage stabilization justifies its use as a stand-alone procedure in lumbar fusion.

\section{PATIENTS AND METHODS}

\section{Patients}

Four patients with degenerative disc disease, all women, had an anterior lumbar fusion with threaded interbody cages. Mean age was 40 (36-44) years. All patients had suffered from long-standing lumbar pain without sciatica with a mean duration of 7 (3-15) years. Three patients were treated with a single-level fusion L5-S1. The fourth patient had a two-level fusion L5-S1 and L4-L5.

\section{External Pedicular Fixation}

Preoperative support for treating the patient with fusion was achieved for all patients by a pain relieving effect registered at temporary stabilization with external pedicular fixation [9]. Pedicular Schanz screws for the external frame [10] were inserted in general anaesthesia with percutaneous technique using an image intensifier for orientation both in the AP and lateral plane. During the same anaesthesia, also with percutaneous technique, tantalum indicators for RSA 
were placed into the bases of the transverse processes and in the spinous process of the vertebrae to be temporarily stabilized by the frame. The lateral masses and the central crest were used in the sacrum. After frame application, the pain relieving effect of the temporary stabilization was assessed during a test period of one week and finished by the extraction of the pedicular screws [11].

\section{Surgery}

Anterior lumbar interbody fusion was performed 2 months after finishing the external fixation test when the soft tissues were fully healed after pedicular screw extraction. At surgery, the anterior part of the disc to be fused was exposed via a retroperitoneal approach. After disc resection and preparation of the end plates, two threaded, cylindrical cages filled with autograft from the anterior iliac crest were inserted in the disc space. The BAK interbody fusion device [12] was used in all cases as a stand-alone procedure with no supplementary instrumentation. Additional tantalum indicators were implanted in the ventral parts of the vertebrae fused (Fig. 1) in order to enlarge the rigid body to be created for the radiostereometric measurement.

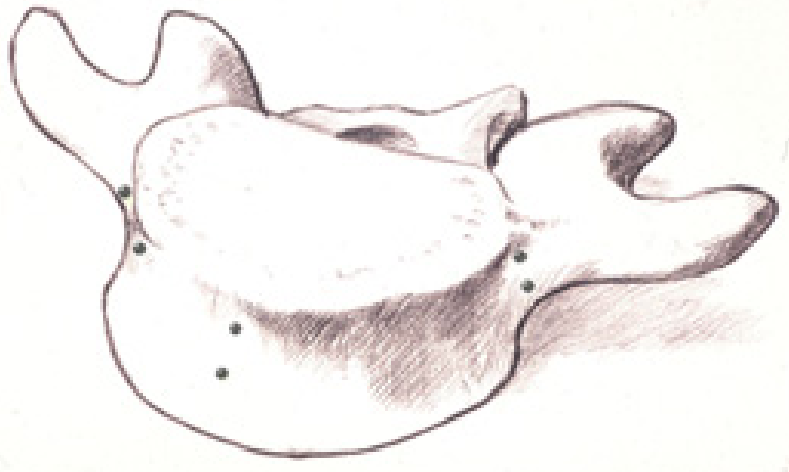

Fig. (1). Additional tantalum indicators applied in the ventral parts of the vertebra during anterior fusion in order to enlarge the rigid body for radiostereometry. (Reprinted with permission from Acta Orthopaedica).

\section{Radiostereometry}

Each patient had a spinal RSA [6] two months after completion of the external fixation test but immediately prior to the definitive fusion procedure. Repeat radiostereometric examinations were performed 1,6 and 12 months after surgery. The RSA set-up was identical at all four occasions. The lumbosacral spine and a combined reference plate and calibration device, with tantalum indicators at known positions in front of the film plane, were exposed simultaneously on two separate films by the use of two 40 degrees angulated roentgen tubes. The translatory movements along the transverse (x), vertical (y) and sagittal (z) axes for the vertebra proximal to the cages relative to the vertebra distally were calculated with the Kinema program for computed data processing according to Selvik [5]. These translatory movements were provided in a standardized manner by the patient moving from supine to a sitting position avoiding any other active mobility provocation in order to reduce the confounding effects of varying pain levels and muscular spasm [13]. The sitting position here means semiflexed hips to allow x-rays to pass above the femoral shafts to the lumbosacral spine.

For determination of the accuracy of RSA in the set-up chosen in this study and also previous ones, minimum significant mobility measurement has been performed and determined to $0.5,0.5$ and $0.7 \mathrm{~mm}$ along the transverse, vertical and sagittal axis according to prior calculations based on double examinations of healed fusions [14]. Translation values below these levels were considered insignificant in this study.

\section{Radiography}

Conventional radiography was included, anteroposterior and lateral view, at follow-up 1, 6 and 12 months after surgery.

\section{RESULTS}

Before surgery, remaining intervertebral mobility was verified for all the five lumbar segments scheduled to be fused (Table 1). Sagittal mobility response, significant in all patients, ranged from $3.1 \mathrm{~mm}$ in patient number 1 to $1.3 \mathrm{~mm}$ in patient number 2, level L4-L5. The corresponding range for vertical translation was from $1.8 \mathrm{~mm}$ in patient number 4 to $0.5 \mathrm{~mm}$ in patient number 1 . No significant transverse translations were registered.

One month after surgery, the intervertebral mobility was fully abolished over 3 fused segments and only small remaining translations along the $\mathrm{z}$-axis were registered for patient number 2, level L5-S1 and for patient number 3 (Table 1). No segment was destabilized by the surgical procedure. One year after surgery, the L5-S1 level of patient number 2 also turned stable and the only remaining mobility was minimal and sagittal seen in patient number 3 .

Table 1. Intervertebral Mobility (mm) Before and Through the Postoperative Course After Anterior Fusion

\begin{tabular}{|c|c|c|c|c|c|c|c|c|c|c|c|c|c|}
\hline \multirow{2}{*}{ Patient Number } & \multirow{2}{*}{ Spinal Level } & \multicolumn{2}{|c|}{ Preoperative Mobility } & \multicolumn{2}{|c|}{ 1 Month After Surgery } & \multicolumn{3}{|c|}{ M Months After Surgery } & \multicolumn{2}{|c|}{ 12 Months After Surgery } \\
\cline { 2 - 15 } & & $\mathbf{X}$ & $\mathbf{Y}$ & $\mathbf{Z}$ & $\mathbf{X}$ & $\mathbf{Y}$ & $\mathbf{Z}$ & $\mathbf{X}$ & $\mathbf{Y}$ & $\mathbf{Z}$ & $\mathbf{X}$ & $\mathbf{Y}$ & $\mathbf{Z}$ \\
\hline \hline 1 & L5-S1 & 0.4 & 0.5 & 3.1 & 0.1 & 0.1 & 0.1 & 0.1 & 0.1 & 0.1 & 0.1 & 0.1 & 0.1 \\
\hline 2 & L5-S1 & 0.1 & 0.9 & 1.4 & 0.1 & 0.1 & 0.8 & 0.1 & 0.1 & 1.1 & 0.1 & 0.1 & 0.4 \\
\hline 2 & L4-L5 & 0.2 & 1.2 & 1.3 & 0.2 & 0.4 & 0.3 & 0.1 & 0.1 & 0.2 & 0.1 & 0.1 & 0.1 \\
\hline 3 & L5-S1 & 0.4 & 1.2 & 2.8 & 0.1 & 0.2 & 0.9 & 0.1 & 0.4 & 1.6 & 0.1 & 0.1 & 0.8 \\
\hline 4 & L5-S1 & 0.1 & 1.8 & 2.0 & 0.1 & 0.1 & 0.4 & 0.1 & 0.2 & 0.2 & 0.1 & 0.1 & 0.1 \\
\hline
\end{tabular}


Conventional radiography 1,6 and 12 months after surgery revealed adequate placement of the interbody cages with no dislocation over time and with no sign of reaction/osteolysis around the implants.

\section{DISCUSSION}

There are several surgical ways to achieve spinal fusion when treating low back pain in degenerative conditions. Three different techniques, posterolateral fusion without instrumentation, posterolateral fusion with posterior instrumentation and posterolateral fusion with posterior instrumentation and interbody fusion, have shown equal clinical outcome at two-year follow-up [15]. The complication rate does however widely favour the uninstrumented posterolateral fusion [16]. This fact seems to have a low impact among the majority of spinal surgeons who prefer alternative techniques. In Sweden, less than 5 percent of the patients fused for degenerative disc disease/segmental pain had an uninstrumented posterolateral fusion according to the reports from the National Spine Register [17]. Posterolateral fusion with posterior instrumentation dominates but also interbody fusion, anterior or posterior, is frequently used.

Interbody cage stabilization as a stand-alone procedure in lumbar spine fusion is questioned and surgeons often recommend the use of a supplementary anterior/posterior instrumentation. Several studies using human cadaveric models analyse the three-dimensional stability putting standardized well-defined load on a specimen [3]. In flexion, the mobility reduction is around 40 percent both for the anterior (ALIF) and posterior (PLIF) approach. In extension, no stabilizing effect is seen neither by ALIF nor PLIF. Mobility response in both axial rotation and lateral bending are reduced with the two types of approach with some advantage for the anterior technique. These mechanical effects are however inconsistent and the mobility with cages is reported sometimes to exceed the normal range of motion before intervention [4]. This potential, undesired destabilization is explained by the resection of the anterior longitudinal ligament included in the ALIF procedure and by the medial facetectomy required for the PLIF.

Radiostereometry brings the possibility to analyse the in vivo conditions induced by interbody cage stabilization. In our study, no sign of initial surgical destabilization was seen. Instead, all five segments had a significant mobility reduction one month after surgery and three were fully stabilized with no residual mobility over the segment. These three segments remained stable over time and a fourth segment turned fully stable after one year. Only in one patient, number 3, a small persisting sagittal translation was seen at the one-year follow-up. In spite of this finding, conventional radiography revealed no dislocation or reaction around the implant (Fig. 2A, B) and has probably limited diagnostic value in this context.

A few facts concerning the study design, which has limitations, can be commented and clarified. The mobility comparison versus the intervertebral mobility of the same segment before surgery strengthens the study result but does, on the other hand, limit the number of patients available for inclusion. The method to provide lumbar mobility at radiostereometry can be questioned by the lack of provocation in extension which from cadaveric studies is the mobility least stabilized by the interbody cages [3] and anterior as well as posterior stand-alone cages have been shown to have an inconsistent stabilization in vitro [4]. Our set-up instead favours the standardization of the examination avoiding active lumbar motion which is a prerequisite when the patients are examined at different occasions with varying pain levels and grade of muscular spasm.

(A)

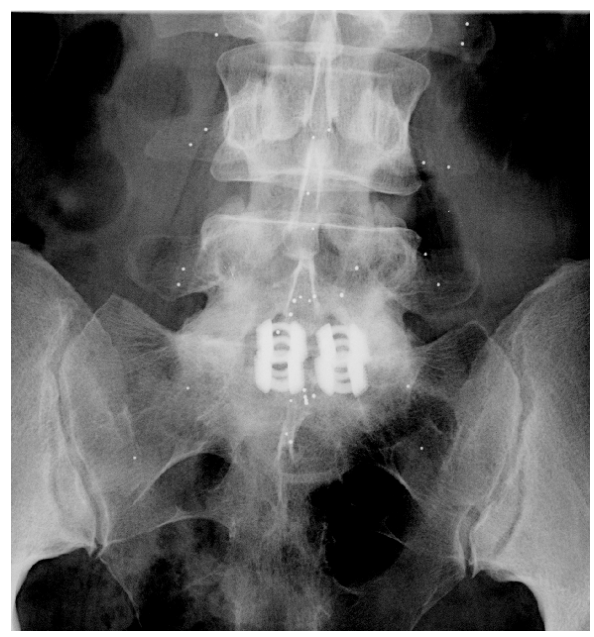

(B)

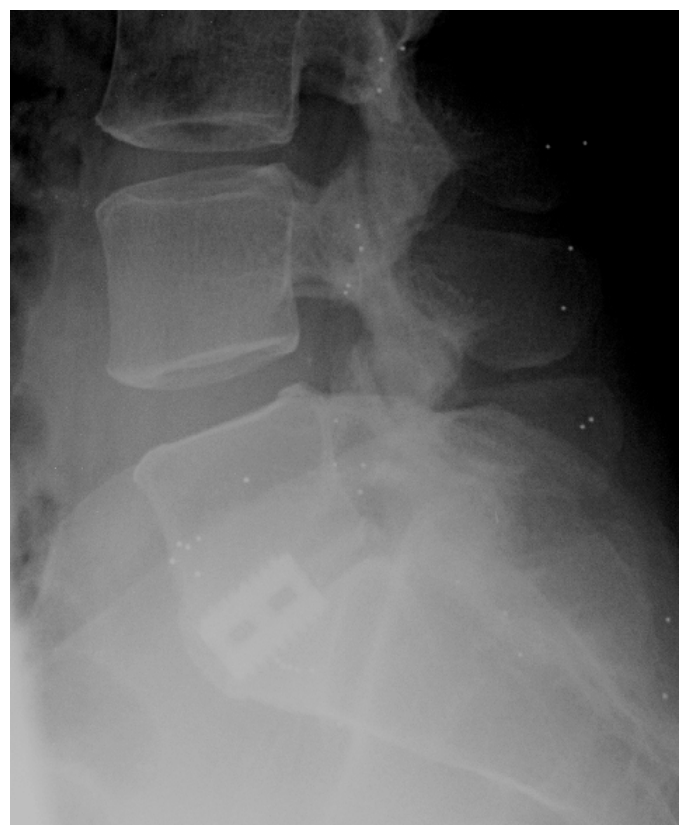

Fig. (2). Patient number 3. Conventional radiography showing no reaction around the implant at one-year follow-up even though minor sagittal mobility persisted over the segment. Tantalum indicators both in the ventral and dorsal parts of the vertebrae aimed to be fused. (A) Anteroposterior view. (B) Lateral view.

In conclusion, our study does not confirm a risk of initial surgical destabilization for the anterior interbody cage fusion. The surgical procedure with cages instead induces an immediate stabilization that brings biomechanical support for the use of the technique as a stand-alone procedure without supplementary instrumentation. Reported high pseudarthrosis rates [2] and specific complications as 
postoperative sexual dysfunction for the anterior procedure [18] are however facts that must be clarified in order to evaluate the anterior interbody cage stabilization as instrument in lumbar spine fusion. The technique must also be compared with the new treatment strategy using disc prosthesis for which initial reports are positive in patients with degenerative disc disorders $[19,20]$. This patient group is however not uniform. Disc height reduction, grade of facet joint arthrosis and preoperative deformity are factors to be studied regarding their influence on which patients benefit from restored segmental mobility by arthroplasty and which patients rather need the locking of mobility induced by fusion. Our study does not demonstrate that anterior standalone cages have a role in lumbar spine fusion but demonstrates initial destabilization after surgery not to be a general phenomenon.

\section{ACKNOWLEDGEMENTS}

This study was supported by grants from the Medical Faculty of the University of Lund, Stiftelsen för bistånd åt vanföra i Skåne, Stiftelsen Tornspiran, The Swedish Society of Medicine (Tryggers Fond) and The Swedish Medical Research Council (No 17X-09509).

\section{REFERENCES}

[1] Kuslich SD, Ulstrom CL, Griffith SL, Ahern JW, Dowdle JD. The Bagby and Kuslich method of lumbar interbody fusion. History, techniques, and 2-year follow-up results of a United States prospective, multicenter trial. Spine 1998; 23: 1267-79.

[2] Nilsson LT, Geijer M, Neumann P, Lind B. The Brantigan anterior lumbar I/F cage. Two years radiological results. Eur Spine J 2001; 10 (Suppl 1): S26.

[3] Oxland TR, Lund T. Biomechanics of stand-alone cages and cages in combination with posterior fixation: a literature review. Eur Spine J 2000; 9 (Suppl 1): S95-S101.

[4] Vishteh AG, Crawford NR, Chamberlain RH, et al. Biomechanical comparison of anterior versus posterior lumbar threaded interbody fusion cages. Spine 2005; 30: 302-10.

[5] Selvik G. Roentgen stereophotogrammetry. A method for the study of the kinematics of the skeletal system. Acta Orthop Scand 1989; 60 (Suppl 232): 1-51.

[6] Johnsson R, Selvik G, Strömqvist B, Sundén G. Mobility of the lower lumbar spine after posterolateral fusion determined by roentgen stereophotogrammetric analysis. Spine 1990; 15: 347-50.
[7] Axelsson P, Johnsson R, Strömqvist B. Radiostereometry in lumbar spine research. Acta Orthop 2006; 77(Suppl 323): 1-42.

[8] Johnsson R, Axelsson P, Gunnarsson G, Strömqvist B. Stability of lumbar fusion with transpedicular fixation determined by roentgen stereophotogrammetric analysis. Spine 1999; 24: 687-90.

[9] Olerud S, Sjöström L, Karlström G, Hamberg M. Spontaneous effect of increased stability of the lower lumbar spine in cases of severe chronic back pain. The answer of an external transpeduncular fixation test. Clin Orthop 1986; 203: 67-74.

[10] Magerl FP. Stabilization of the lower thoracic and lumbar spine with external skeletal fixation. Clin Orthop 1984; 189: 125-41.

[11] Axelsson P, Johnsson R, Strömqvist B, Andréasson H. External pedicular fixation of the lumbar spine. Outcome evaluation by functional tests. J Spin Disord 1999; 12: 147-50.

[12] Nibu K, Panjabi MM, Oxland T, Cholewicki J. Multidirectional stabilizing potential of BAK interbody spinal fusion system for anterior surgery. J Spin Disord 1997; 10: 357-62.

[13] Axelsson P, Karlsson BS. Standardized provocation of lumbar spine mobility. Three methods compared by radiostereometric analysis. Spine 2005; 30: 792-7.

[14] Johnsson R, Strömqvist B, Aspenberg P. Randomized radiostereometric study comparing osteogenic protein-1 (BMP-7) and autograft bone in human noninstrumented posterolateral lumbar fusion. Spine 2002; 27: 2654-61.

[15] Fritzell P, Hägg O, Wessberg P, Nordwall A. Chronic low back pain and fusion - a comparison of three surgical techniques. A prospective multicenter randomized study from the Swedish Lumbar Spine Study Group. Spine 2002; 27: 1131-41

[16] Fritzell P, Hägg O, Nordwall A. Complications in lumbar fusion surgery for chronic low back pain: comparison of three surgical techniques used in a prospective randomized study. A report from the Swedish Lumbar Spine Study Group. Eur Spine J 2003; 12: 178-89.

[17] Strömqvist B, Fritzell P, Hägg O, Jönsson B. One-year report from the Swedish National Spine Register. Swedish Society of Spinal Surgeons. Acta Orthop 2005; 76 (Suppl 319): 1-24.

[18] Hägg O, Fritzell P, Nordwall A and the Swedish Lumbar Spine Study Group. Sexual function after surgery for chronic low back pain. Eur Spine J 2003; 12 (Suppl 1): S17-S18.

[19] Blumenthal S, McAfee PC, Guyer RD, et al. A prospective, randomized, multicenter Food and Drug Administration investigational device exemption study of lumbar total disc replacement with the CHARITÉ artificial disc versus lumbar fusion. Part I: Evaluation of clinical outcomes. Spine 2005; 14: $1565-75$.

[20] McAfee PC, Cunningham B, Holsapple G, et al. A prospective, randomized, multicenter Food and Drug Administration investigational device exemption study of lumbar total disc replacement with the CHARITÉ artificial disc versus lumbar fusion. Part II: Evaluation of radiographic outcomes and correlation of surgical technique accuracy with clinical outcomes. Spine 2005; 30: $1576-83$

(C) Axelsson et al.; Licensee Bentham Open.

This is an open access article licensed under the terms of the Creative Commons Attribution Non-Commercial License (http: //creativecommons.org/licenses/ by-nc/3.0/) which permits unrestricted, non-commercial use, distribution and reproduction in any medium, provided the work is properly cited. 